This manuscript has been submitted for publication to Society of Exploration Geophysicists (SEG). Please note that, this manuscript is a non-peer reviewed preprint. Subsequent versions of this manuscript may have different content and more examples. If accepted, the final version of this manuscript will be available via this webpage. Please feel free to contact the authors; we welcome feedback.

\title{
SymAE: an autoencoder with embedded physical symmetries for passive time-lapse monitoring
}

\author{
Pawan Bharadwaj ${ }^{* 1}$, Matt $\mathrm{Li}^{\dagger 1}$ and Laurent Demanet ${ }^{\ddagger 1}$ \\ ${ }^{1}$ Department of Mathematics \& Earth Resources Laboratory, Massachusetts Institute of \\ Technology
}

April 12, 2020

We introduce SymAE, an auto-encoder architecture that learns to separate multichannel passive-seismic datasets into qualitatively interpretable components: one component corresponds to path-specific effects associated with subsurface properties while the other component corresponds to the spectral signature of the passive sources. This information is represented by two latent codes produced by our encoder. The novelty that enables SymAE to achieve this separation lies with the physical symmetries that are directly embedded into the architectural design of the encoder. These symmetries impose that 1 . the output of the source-specific encoder is indifferent to the ordering of the receivers; and 2. the output of the path-specific encoder is indifferent to the source signatures. Our numerical experiments demonstrate that this is sufficient for achieving the intended separation.

The ability to qualitatively distinguish between source- and path- induced effects plays a critical role for time-lapse monitoring of visco-acoustic subsurface models where data is generated from induced passive seismic sources e.g., during $\mathrm{CO} 2$ injection or hydraulic fracturing. Here the problem suffers from inherent ambiguities in whether the time-lapse changes in the data should be attributed to subsurface changes such as $\mathrm{P}$-wave velocity, mass density, and seismic quality factor (i.e., path effects) or because of difficulties in physicallyreproducing the source wavelet (i.e. source effects). SymAE resolves these ambiguities by construction and enables reliable subsurface monitoring in these settings. We provide numerical results to show that we can accurately detect changes arising from both effects.

\section{Introduction}

During time-lapse monitoring of subsurface changes, e.g. due to oil/gas production (Lumley, 2001) and carbon-dioxide flooding of reservoirs (Wang et al., 1996), the principle challenge is in maintaining similar acquisition-related parameters across the baseline and monitor experiments. If this repeatability between experiments is achieved then any changes observed in the recorded data can be solely attributed to changes that appear in the path effects (i.e. the subsurface properties). However, in practice, uncontrollable factors involved in the acquisition prevent repeatability. In this note we consider a scenario where repeatability is impeded by lack of control over the source, and consequently the input signatures differ between timelapse experiments conducted across different timescales. This setup is motivated, in part, by Pevzner et al. (2011) which reports that the ground-coupling and source-type effects dominate all other effects when investigating time-lapse differences in the reservoir for carbon sequestration. Similar difficulties are reported in passive time-lapse monitoring where the data is recorded from micro-earthquakes from which source repeatability is physically unachievable.

Nevertheless, even when repeatibility is not guaranteed, these experiments routinely record an abundance of data (with a multitude of source signatures) at multiple receivers. For example, a number of micro-earthquakes

*pawbz@mit.edu

†mtcli@mit.edu

‡laurent@math.mit.edu 


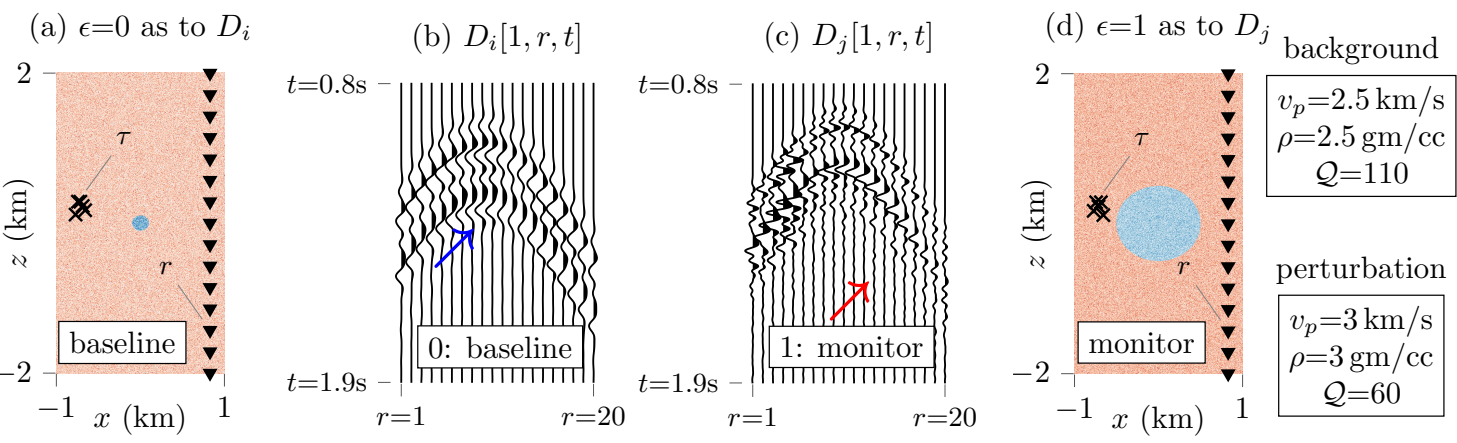

Figure 1: Passive-seismic monitoring captures an expanding circular perturbation during (a) baseline and (d) monitor experiments. Input datapoints $\left[D_{i}\right]$ to SymAE contain records due to multiple source instances $\tau$ that are marked using crosses. Recorded data, for $\tau=1$, during (b) baseline and (c) monitor experiments show hints of time-lapse path-effect changes (marked using red and blue arrows), along with source-signature variability. The observation-well receivers $(\approx 10$ wavelengths away) are marked with triangles.

with different signatures occur during injection/production - the intention here is to harvest the records at permanently deployed receiver stations to monitor saturation changes in a petroleum reservoir (Maxwell et al., 2010). In principle advanced signal processing methods can be applied to these massive datasets to detect whether time-lapse changes arise due to source effects or when changes arise due to path effects. Blind deconvolution can produce this separation, but existing algorithms depend heavily on known low-dimensional parameterizations for the filter (here, the path effect) and the signal (here, the source) (Ahmed et al., 2015). These assumptions are difficult to obtain in real-world settings. Instead, in this work we echew this approach and leverage the massive datasets to achieve this goal via data-driven representation learning.

We propose an extension of the auto-encoder (Kramer, 1991; Goodfellow et al., 2016), an unsupervised machine learning model that is trained to provide a compressed representation of an input set of "datapoints" $\left[D_{i}\right]$. In our setting these datapoint correspond to the passive-seismic data. Functionally autoencoders are comprised of two components: an encoder Enc that maps datapoint $D_{i}$ into latent code $H_{i}=\operatorname{Enc}\left(D_{i}\right)$, and a decoder Dec that attempts reconstruct $D_{i}$ from the code. Both functions Enc and Dec are determined by minimizing the reconstruction loss

$$
\text { Enc, Dec }=\underset{\text { Enc, Dec }}{\arg \min } \sum_{i}\left\|D_{i}-\operatorname{Dec}\left(\operatorname{Enc}\left(D_{i}\right)\right)\right\|^{2}
$$

over a training dataset. When Enc and Dec are constrained to be linear operators, this program recovers principle-component analysis; hence the output of the encoder (i.e. the latent code) is analogous to the singular values, while the encoder plays the role of the left singular vectors of the training dataset. Intuitively the role of Dec is to reconstruct the source and path effects in the time-series data based on the latent representation.

When non-linear parameterizations are used for both Enc and Dec the resulting latent codes are difficult to physically interpret; the latent representation no longer describe the geometry of the datasets using linear subspaces (Klys et al., 2018). More critically for our specific applications, a direct application of the traditional autoencoder will not ensure that the source and path effects are encoded into separate components (dimensions) in the latent space. Although regularization functionals can be supplemented to the square-loss function to promote such disentangled representations (Chen et al., 2018) these approaches are typically ad-hoc without any consideration of the provenance of the data.

In this note we achieve separability by modifying the architecture to exploit physical symmetries that we expect to observe in the data, rather than modifying the loss function. The key idea is that we separate Enc into two encoders GEnc and WEnc that both impose complementary symmetries, i.e. the symmetry imposed by one encoder is not imposed by the other. This ensures that the codes $W$ and $G$ produced by these functions correspond to either the source or the path effects, respectively, but does not contain information about both. We refer to an auto-encoder architecture with these symmetric functions embedded in its encoder as SymAE. We mention here related work that embed symmetries and invariances into neural network architectures; Mattheakis et al. (2019) embedded even/odd symmetry of a function and energy conservation into a neural network by adding special $h u b$ layers; Cohen et al. (2019) propose gauge equivariant CNN layers to capture rotational symmetry; Greydanus et al. (2019) structures their networks following a Hamiltonian in order to learn physically conserved quantities and symmetries. 
Figure 2: Each datapoint, indexed as $D[\tau, r, t]$, has a unique experiment label $\epsilon$ e.g., (a) $D_{i}$ and (b) $D_{j}$ contain data recorded during experiments 0 (baseline) and 1, respectively. Some time-lapse path-effect changes are marked using red and blue arrows.

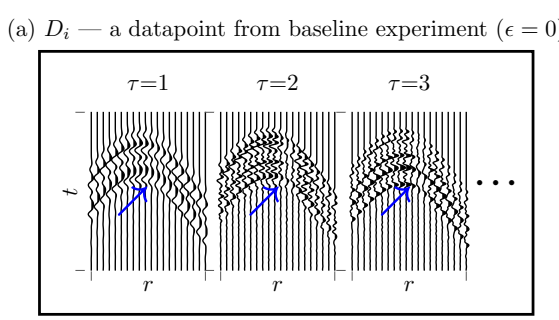

(b) $D_{j}$ - a datapoint from monitor experiment $(\epsilon=1)$

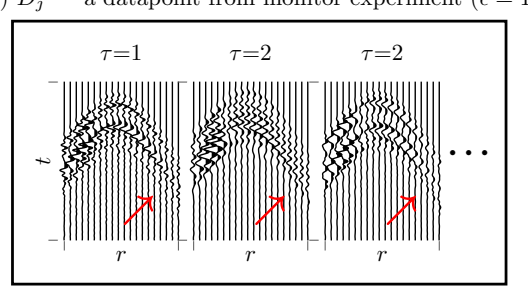

WEnc: source encoder/ path-effect annihilator

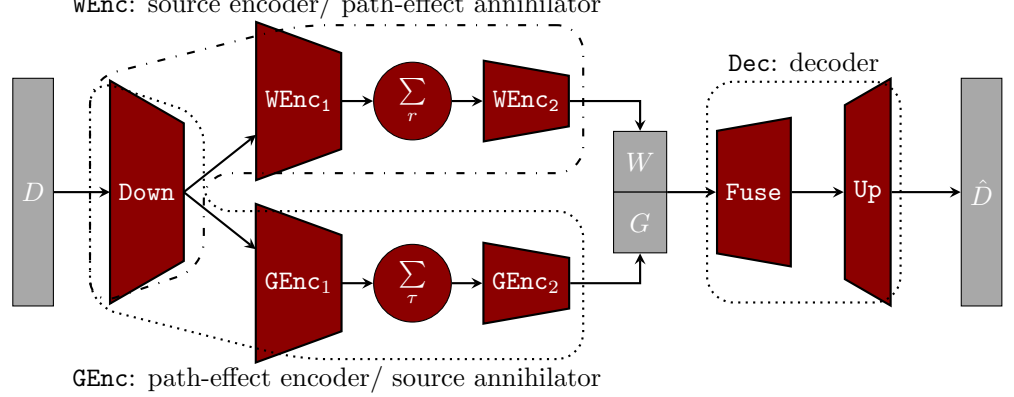

Figure 3: SymAE architecture recognizes that the source-signature encoder WEnc is symmetric w.r.t. the order of receivers in, and the path-effect encoder GEnc is symmetric w.r.t. the order to instances in the input datapoint $D$. Embedding these symmetries results in a latent space representation with disentangled source $W$ and path $G$ effects. Non-linear down- and up-sampling along the recordtime dimension is achieved using networks Down and Up.

\section{Time Lapse Monitoring}

Applying SymAE to time-lapse monitoring amounts to monitoring for changes in $G$, which represents the complex path information in the passive-seismic records. When compared against existing time-lapse monitoring approaches SymAE is also more robust to real-world artifacts since it is trained with massive datasets. For instance, typically traditional methods for passive-seismic data rely on assuming time-stationary source distributions (Garnier and Papanicolaou, 2016). However, a transient source amplitude spectrum is often observed in the ambient seismic field and these spectrum fluctuations may interfere with the time-lapse subsurface changes of interest. As a result, traditional methods primarily extract kinematic information from stacking several finite-length cross-correlated records (Nakata and Snieder, 2012; Mordret et al., 2014). De Ridder et al. (2014) discard the recorded amplitude spectrum in order to reliably pick group travel times after cross-correlation. In contrast SymAE doesn't require a time stationary source and can monitor changes in the full wavefield irrespective of the source spectrum.

We demonstrate these capabilities with numerical experiments to show that SymAE identifies time-lapse changes in the $Q$-factor attenuation at different recorded frequencies. Winkler and Nur (1979) notes that timelapse saturation changes on account of variations in the intrinsic attenuation of rocks is more than an order of magnitude greater than changes induced by variations in velocity. In order to disentangle the source and path effects, SymAE associates the path effects to the dissimilarities among the arrivals recorded at multiple receiver stations, and the remaining similarities are identified as the source. Existing signal processing methods that extract the time-lapse attenuation changes also utilize the relative changes in the amplitude and phase spectra among various arrivals in the data (Rickett, 2007; Shabelansky et al., 2015). Bharadwaj et al. (2019) proposed a blind-deconvolution algorithm, which also extracts dissimilarities in the multichannel records using focusing constraints, but assumes that the path effects are front-loaded in time.

We use $\epsilon$ to denote a passive-source experiment label, where the baseline (with label $\epsilon=0$ ) is followed by monitoring experiments. Typically, such experiments are performed once every few months to monitor the changes in the medium properties. The duration of each experiment is in the order of hours or days, in which changes in the medium are negligible. During each experiment, various arbitrary source signatures or instances, denoted by $[w(t ; \tau)]_{\tau=1,2, \ldots}$, generate waves through the medium. Here, we use $t$ to denote the record or fast time which is usually in the order of seconds. We write the wave-equation as $\mathcal{L}(\epsilon) d(\mathbf{x}, t)=w(t ; \tau) \delta\left(\mathbf{x}-\mathbf{x}_{0}\right)$, where $(\mathbf{x}, t) \in \mathbb{R}^{3} \times \mathbb{R}$ where $\mathcal{L}(\epsilon)$ denotes the wave operator with medium parameters corresponding to the experiment $\epsilon$. The recorded wavefield at a location $\mathbf{x}_{r}$ for instance $\tau$ during experiment $\epsilon$ is given by a temporal convolution:

$$
d\left(t, \mathbf{x}_{r} ; \tau, \epsilon\right)=\int w\left(t-t_{1} ; \tau\right) g\left(t_{1}, \mathbf{x}_{r} ; \epsilon\right) \mathrm{d} t_{1}
$$

The time-dependent Green's function $g(t, \mathbf{x} ; \epsilon)$ represents the path effects in the medium during $\epsilon$, due to an impulsive source at a fixed location $\mathbf{x}_{0}$. The following examples illustrate how instances are recorded during a given experiment: 
(a) $\hat{D}_{i}[1, r, t]$

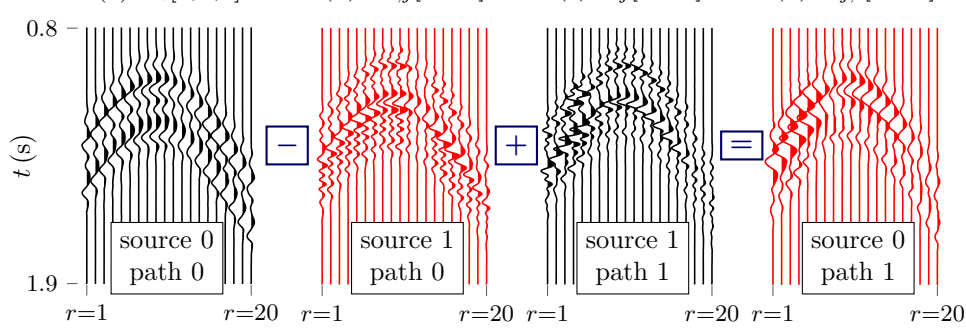

Figure 4: An illustration where the source and path effects in passive-seismic data are manipulated using vector algebra in SymAE's latent space. Hybrid datapoints, (b) and (c) plotted in red, are generated by exchanging either the source- or path-related latent codes between original datapoints. Notice that the hybrid datapoint $\hat{D}_{j, i}$ contains the source signature of $D_{i}$ and the path effects (blue and red arrows in Fig. 1) of $D_{j}$. $\begin{array}{llll}\text { (a) } \epsilon=0 & \text { (b) } \epsilon=.25 \quad \text { (c) } \epsilon=.5 \quad \text { (d) } \epsilon=.75 \quad(\mathrm{e}) \epsilon=1\end{array}$

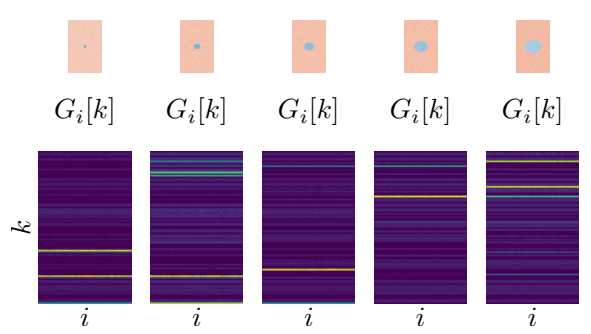

Figure 5: A demonstration that the encoder GEnc of SymAE outputs a code $G$ that is invariant to the source effects in $D_{i}$. Here, the datapoints $\left[D_{i}\right]$ are grouped in (a)-(e) based on the experiment index $\epsilon$. The expanding circular perturbation for each $\epsilon$ is also plotted.

- For microseimic monitoring (Eyre et al., 2019; White and Foxall, 2016), earthquakes with different signatures but with roughly the same hypocenter $\mathbf{x}_{0}$, i.e., repeating earthquakes that rupture the same fault zone, generate required instances $w(t ; \tau)$ (see Fig. 1a).

- For monitoring using ambient noise (Mordret et al., 2014), we assume a stationary spatial source distribution in a particular time interval, the recorded noise can be divided into several smaller time windows to produce instances.

In this paper we focus on the microseismic application with an active fault zone in all the experiments. An illustrative visco-acoustic time-lapse example with an expanding circular perturbation is presented in Fig. 1. We follow the viscous materials model of Carcione et al. (1988) where components such as springs and dashpots form an array of standard linear solids to approximate a viscosity model with nearly constant quality factor $\mathcal{Q}$ over the seismic frequency range (Robertsson et al., 1994). Each instance $w(t ; \tau)$ is generated by convolving a Ricker wavelet whose dominant frequency is sampled from $\{10,12.5, \ldots, 20\} \mathrm{Hz}$, with a random time series (of $0.4 \mathrm{~s}$ duration) sampled from a standard normal distribution. The modeled records using these instances during baseline $(\epsilon=0)$ and monitor $(\epsilon=1)$ experiments are plotted in Figs. 1b and 1c, respectively. The path effects, e.g., the attenuation of higher frequencies travelling through the circular perturbation with lower $\mathcal{Q}$, in these plots are indicated using red and blue arrows. For this example we consider a total of five experiments with $\epsilon \in\{0,0.25, \ldots, 1\}$, and modeled data using 1600 instances per experiment.

The data is arranged into an array of $n_{D}$ datapoints, denoted by $\left[D_{i}\right]_{i=1: n_{D}}$, where each datapoint $D_{i}$ is a multidimensional array indexed as $D_{i}[\tau, r, t]$. Following the above discussion, $\tau$ denotes the instance index running from 0 to $n_{\tau}, r$ denotes receiver index running from 1 to $n_{r}$, and $t$ denotes the fast-time index running from 0 to $n_{t}$. Each datapoint has an associated experiment label i.e., it is constructed using data measured during a specific experiment. For the visco-acoustic example we construct a total of $n_{D}=10^{4}$ datapoints with $n_{\tau}=20, n_{r}=20$ and $n_{t}=729$ - they are plotted for $\epsilon=0$ and $\epsilon=1$ in Figs. 2a and 2b, respectively. To reiterate, our goal is to extract the time-lapse differences in the path-effects between any two given datapoints, irrespective of the variability in source instances.

\section{SymAE}

SymAE's network architecture is depicted in Fig. 3, and the Tensorflow (Abadi et al., 2016) code snippets in Lsts. 1-6 provide further details on the implementation used for our visco-acoustic numerical examples. At a high level SymAE relies on the special encoder structure $\operatorname{Enc}\left(D_{i}\right)=\left[\operatorname{GEnc}\left(D_{i}\right) ; \operatorname{WEnc}\left(D_{i}\right)\right]$ to partition the latent code $Z$ into physically-interpretable components. In other words, the network represents passiveseismic datapoints using a structured latent code $H=[G ; W]$ where the sub-components $G=\operatorname{GEnc}\left(D_{i}\right)$ contain information on the path effects in $D_{i}$ while the sub-components $W=\operatorname{WEnc}\left(D_{i}\right)$ complement this with information about the source-effects in $D_{i}$. Here, $[A ; B]$ denotes a vertical concatenation of two arrays $A$ and $B$. Evidently this separation critically relies on the inability of encoder WEnc (resp. GEnc) to encode path information (resp. source information). The key insight is that the "cross-talk" between the encodings $G$ and $W$ can be discouraged by enforcing the encoders to comply with the following physical symmetries.

- The source-signature information is symmetric w.r.t. (i.e. independent of) the labeling of the receivers in $D_{i}$. Thus, WEnc should be invariant to permutations, which are obtained using a function $\Pi$, in the receiver 
dimension:

$$
W_{i}=\operatorname{WEnc}\left(D_{i}\right)=\operatorname{WEnc}\left(D_{i}\left[:, \Pi\left(1: n_{r}\right),:\right]\right) .
$$

- Similarly, the path-effects are symmetric w.r.t the order of instances in $D_{i}$. Hence GEnc should reflect similar symmetries

$$
G_{i}=\operatorname{GEnc}\left(X_{i}\right)=\operatorname{GEnc}\left(X_{i}\left[\Pi\left(1: n_{\tau}\right),:,:\right]\right) .
$$

The above equations use an indexing notation where e.g., $D_{i}[:, 1,:]$ denotes a 2 -D slice of the 3 -D array $D_{i}$ corresponding to the first receiver, and $D_{i}[\Pi(1: 3),:,:]$ denotes a subarray extracted after permuting the first three instances. SymAE uses permutation-invariant network architectures following (Zaheer et al., 2017) which provide universal approximation guarantees for symmetric functions. We now describe an architecture for the path-effect encoder GEnc; the architecture of the source-effect encoder WEnc proceeds analogously. Following Fig. 3 the seismic data $D_{i}$ is first downsampled along the $t$ dimension by distributing a non-linear transformation Down among the $\tau$ and $r$ indices. This produces an array of downsampled records with size $n_{\tau} \times n_{r}:$

$$
D_{i}^{\downarrow}=\left[\operatorname{Down}\left(D_{i}[\tau, r,:]\right)\right]_{\tau=1: n_{\tau}, r=1: n_{r}} .
$$

The data due to each source instance are then transformed using GEnc $_{1}$ and summed along the instance dimension. This output is processed by $\mathrm{GEnc}_{2}$ :

$$
G_{i}=\operatorname{GEnc}_{2}\left(\sum_{\tau}\left(\left[\operatorname{GEnc}_{1}\left(D_{i}^{\downarrow}[\tau,:]\right)\right]_{\tau=1: n_{\tau}}\right)\right) .
$$

This comprises of the network architecture of GEnc. In practice the functions Down, GEnc ${ }_{1}$ and GEnc are $_{2}$ parametrized by universal approximators (e.g. compositions of fully connected layers, convolutional layers, etc.), see Lsts. 2, 3 and 4 for details. We emphasize that the key observation in eq. 6 is that the summation of the transformed instances $\left[\operatorname{GEnc}_{1}\left(D_{i}^{\downarrow}[\tau,:]\right)\right]$ is symmetric with respect to the order of its arguments. This ensures that the desired symmetry (eq. 4) is achieved. Alternatively the summation can be replaced other symmetric function such as the max function - we refer to Ilse et al. (2018) for a review of various permutation-invariant architectures.

The SymAE decoder Dec is trained concurrently with WEnc and GEnc to reconstruct the original datapoint from the latent codes $W$ and $G$ by minimizing eq. 1. A crucial component of Dec is the function Fuse which fuses $G$ with several chunks of $W$ (see Lst. 5), before non-linear upsampling with Up along the temporal dimension (see Lst. 6; similar to Down):

$$
\hat{D}^{\downarrow}=\operatorname{Fuse}([G ; W]) ; \quad \hat{D}=\operatorname{Up}\left(\hat{D}^{\downarrow}\right)=\operatorname{Dec}([G ; W]) .
$$

For the visco-acoustic example we achieve less than 0.01 in the normalized mean-squared error between the true $D$ and reconstructed $\hat{D}$ datapoints for both training and testing. The reconstructions of the original instances in Figs. 1b and 1c are plotted in Figs. 4a and 4c, respectively.

\subsection{Similarity}

Finally, note that embedding the aforementioned symmetries means that WEnc is restricted to encode only the similarity among the receivers (resulting in $W$ ), and GEnc is restricted to encode the similarity among the instances (resulting in $G$ ). Thus SymAE requires sufficient dissimilarity along the instance and receiver dimensions for each datapoint in order to achieve the desired structuring of the latent space.

\subsection{Hybrid Datapoints}

Once SymAE learns a representation with disentangled source and path effects, hybrid datapoints can be generated by swapping these effects between any two datapoints. For example, a hybrid datapoint generated using with path information in $D_{i}$ and instances in $D_{j}$ is given by:

$$
\hat{D}_{i, j}=\operatorname{Dec}\left(\left[\operatorname{GEnc}\left(D_{i}\right) ; \operatorname{WEnc}\left(D_{j}\right)\right]\right),
$$

as plotted in Fig. 4b. In a similar manner, the source and path effects in the datapoints can be manipulated individually in the latent space - this is illustrated in the Fig. 4, where a hybrid datapoint $D_{j, i}$ is generated using vector algebra in the latent space. Consequently, the differences between $D_{i}$ and $D_{j, i}$ can be attributed to time-lapse changes in the path effects between the experiments corresponding to the $i$ th and $j$ th datapoints. 


\subsection{Monitoring}

In order to justify that a reliable time-lapse monitoring of the subsurface properties is possible with SymAE, we show that the path-effect code $G$ is indifferent to the source instances for a given $\epsilon$. In Fig $5, G=\operatorname{GEnc}\left(X_{i}\right)$ is plotted for every $i$, after grouping the datapoints based on the experiment $\epsilon$ - notice that $G$ is invariant to the source information.

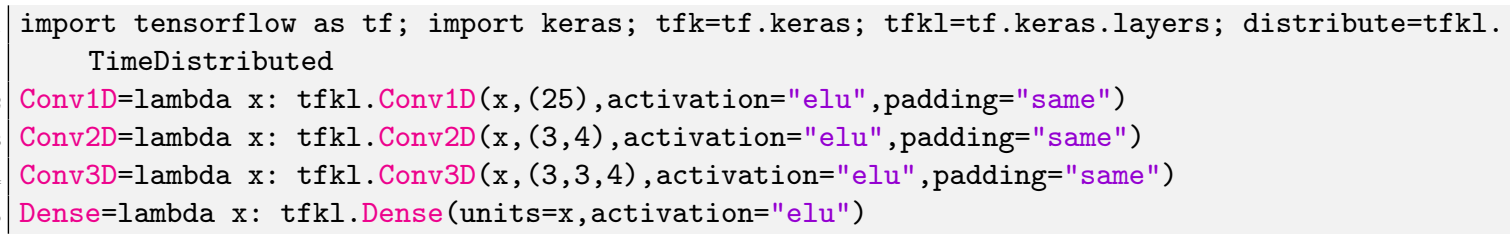

Listing 1: Layers in SymAE's architecture.

$D=$ tfk. Input ( $\left.\operatorname{shape}=\left(n_{\tau}, n_{r}, n_{t}, 1\right)\right)$ \# input datapoint

$D^{\downarrow}=\mathrm{tfkl}$. Reshape (target_shape $\left.=\left(\left(n_{\tau} \times n_{r}, n_{t}, 1\right)\right)\right)(D)$

$D^{\downarrow}=\operatorname{distribute}(\operatorname{Conv1D}(8))\left(D^{\downarrow}\right) ; D^{\downarrow}=\operatorname{distribute}(\operatorname{Conv1D}(8))\left(D^{\downarrow}\right)$

$D^{\downarrow}=$ distribute $\left(\mathrm{tfkl}\right.$. MaxPool1D (pool_size=(3))) $\left(D^{\downarrow}\right)$

$D^{\downarrow}=\operatorname{distribute}(\operatorname{Conv1D}(8))\left(D^{\downarrow}\right) ; D^{\downarrow}=\operatorname{distribute}(\operatorname{Conv1D}(1))\left(D^{\downarrow}\right)$

$n_{t}^{\downarrow}=D^{\downarrow}$.get_shape () [2] ; $D^{\downarrow}=\mathrm{tfkl}$. Reshape (target_shape= $\left.\left(\left(n_{\tau}, n_{r}, n_{t}^{d}, 1\right)\right)\right)\left(D^{\downarrow}\right)$

$D^{\downarrow}=$ tfkl. BatchNormalization() $\left(D^{\downarrow}\right)$

Listing 2: Non-linear downsampling along $t$ dimension.

$G=\operatorname{distribute}(\operatorname{Conv2D}(64))\left(D^{\downarrow}\right) ; G=\operatorname{distribute}(\operatorname{Conv2D}(64))(G)$ \# GEnc 1

$G=$ distribute $(t f k l$.MaxPool2D (pool_size= $(2,4)))(G)$ \# GEnc 1

$G=$ distribute (Conv2D (64)) $(G) ; G=\operatorname{distribute(Conv2D(64))~}(G)$ \# GEnc 1

$G=$ distribute (tfkl.MaxPool2D (pool_size= $(2,4)))(G)$ \# GEnc 1

$G=\mathrm{tfkl}$. AveragePooling3D $\left(\left(n_{\tau}, 1,1\right),\left(n_{\tau}, 1,1\right)\right)(G)$ \# pool over $\tau$

$G=$ tf .squeeze ( $G$, axis $=1) ; G=\operatorname{tfkl}$. BatchNormalization() $(G)$

$G=\operatorname{Conv2D}(64)(G) ; G=\operatorname{Conv2D}(64)(G) \#$ GEnc $_{2}$

$G=\mathrm{tfkl}$.MaxPool2D (pool_size $=(2,4))(G) \#$ GEnc $_{2}$

$G=\operatorname{Conv2D}(64)(G) ; G=\operatorname{Conv2D}(64)(G) \#$ GEnc $_{2}$

$G=\mathrm{tfkl}$. MaxPool2D (pool_size $=(2,4))(G) \# \mathrm{GEnc}_{2}$

$G=\operatorname{tfkl}$. Flatten ()$(G) ; \operatorname{GEnc}=t f \cdot \operatorname{keras} \cdot \operatorname{Model}(D, G)$

Listing 3: $G$ is invariant to permutations in $\tau$ dimension.

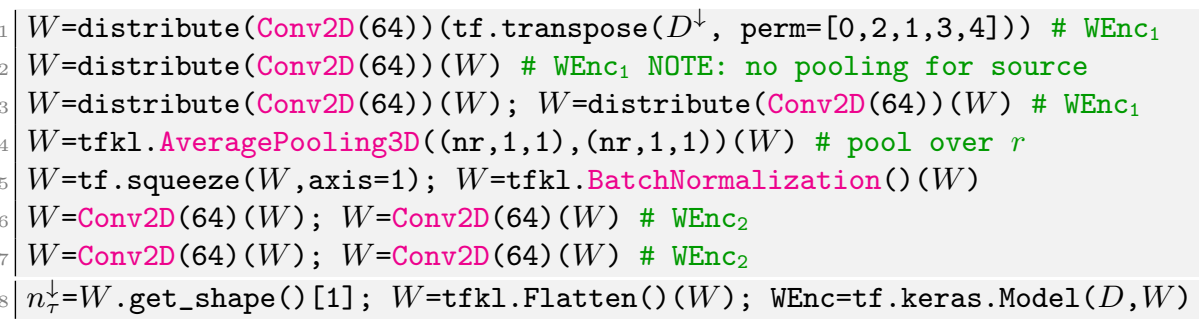

Listing 4: $W$ is invariant to permutations in $r$ dimension.

| $H=$ tf .keras. Input (shape $=\left(G \cdot \operatorname{get} \_\right.$shape ()$[1]+W \cdot \operatorname{get}$ shape () [1] ) )

$\hat{G}, \hat{W}=$ tf .split (decoder_input, [G.getshape () [1] , $W \cdot \operatorname{get}$ shape () [1] ], axis=1)

$\hat{W}=\mathrm{tfkl}$. Reshape $\left(\right.$ target_shape $\left.=\left(n_{\tau}^{\downarrow}, n_{t}^{\downarrow}\right)\right)(\hat{W})$

$\hat{G}=$ tfkl.RepeatVector $\left(n_{\tau}^{\downarrow}\right)(\hat{G})$ \# repeat for each $\tau$

$\hat{G}=\mathrm{tfkl}$. Reshape (target_shape $=\left(n_{\tau}^{\downarrow}, G \cdot \operatorname{get}\right.$ shape () $\left.\left.[1]\right)\right)(\hat{G})$

$\hat{D}^{\downarrow}=\operatorname{tfk} l$. concatenate $([\hat{G}, \hat{W}]$, axis $=2)$

$\hat{D}^{\downarrow}=$ distribute (Dense $\left.\left(\left(\left(n_{r} / / 2\right) \times\left(n_{t}^{\downarrow} / / 3\right)\right)\right)\right)\left(\hat{D}^{\downarrow}\right)$ \# Fuse

$\hat{D}^{\downarrow}=$ distribute (Dense $\left.\left(\left(\left(n_{r} / / 2\right) \times\left(n_{t}^{\downarrow} / / 3\right)\right)\right)\right)\left(\hat{D}^{\downarrow}\right)$ \# Fuse

$\hat{D}^{\downarrow}=$ distribute $\left(\operatorname{tfkl}\right.$. Reshape $\left(\right.$ target_shape $\left.\left.=\left(\left(n_{r} / / 2\right),\left(n_{t}^{\downarrow} / / 3\right), 1\right)\right)\right)\left(\hat{D}^{\downarrow}\right)$

$\hat{D}^{\downarrow}=\operatorname{Conv3D}(64)\left(\hat{D}^{\downarrow}\right) ; \hat{D}^{\downarrow}=$ tfk. layers. UpSampling3D $(\operatorname{size}=(1,2,3))\left(\hat{D}^{\downarrow}\right)$

$\hat{D}^{\downarrow}=\operatorname{Conv3D}(64)\left(\hat{D}^{\downarrow}\right) ; \hat{D}^{\downarrow}=\operatorname{Conv3D}(64)\left(\hat{D}^{\downarrow}\right)$

${ }_{2} \mid \hat{D}^{\downarrow}=\operatorname{Conv3D}(1)\left(\hat{D}^{\downarrow}\right.$, activation=none); $\hat{D}^{\downarrow}=\operatorname{tfkl}$. BatchNormalization ()$\left(\hat{D}^{\downarrow}\right)$

Listing 5: Fuse latent codes $G$ and $W$.

\footnotetext{
$\hat{D}=\mathrm{tfkl}$. Reshape (target_shape $\left.=\left(\left(n_{\tau} \times n_{r}, n_{t}^{\downarrow}, 1\right)\right)\right)\left(\hat{D}^{\downarrow}\right)$

$\hat{D}=\operatorname{distribute}(\operatorname{Conv1D}(8))(\hat{D})$

$\hat{D}=$ distribute $($ tfkl. UpSampling1D $(\operatorname{size}=(3)))(\hat{D})$
} 


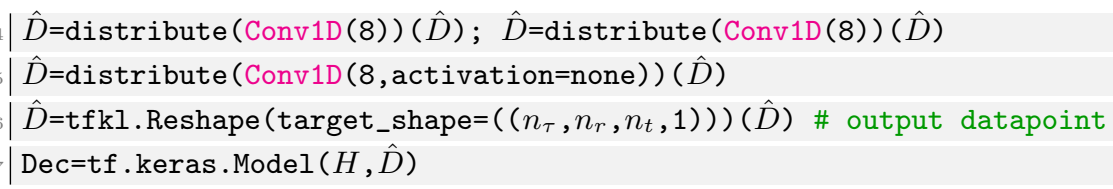

Listing 6: Non-linear upsampling along the $t$ dimension.

\section{Conclusions}

We have presented SymAE, a novel autoencoder for learning the representation of passive-seismic data, where the source and path effects are disentangled. As this structuring of the latent representation is difficult to achieve with traditional autoencoders, we embedded physical symmetries into the encoder architecture such that certain dimensions of the latent code are designed to be indifferent to the ordering of the receivers, therefore containing the source information; and the remaining dimensions are indifferent to instances of the Green's function, therefore containing path information. SymAE's data-driven representation learning can leverage the large passive-seismic datasets to perform an accurate subsurface monitoring.

\section{Acknowledgements}

The authors thank Total SA for support.

\section{References}

Abadi, M., A. Agarwal, P. Barham, E. Brevdo, Z. Chen, C. Citro, G. S. Corrado, A. Davis, J. Dean, M. Devin, S. Ghemawat, I. Goodfellow, A. Harp, G. Irving, M. Isard, Y. Jia, R. Jozefowicz, L. Kaiser, M. Kudlur, J. Levenberg, D. Mane, R. Monga, S. Moore, D. Murray, C. Olah, M. Schuster, J. Shlens, B. Steiner, I. Sutskever, K. Talwar, P. Tucker, V. Vanhoucke, V. Vasudevan, F. Viegas, O. Vinyals, P. Warden, M. Wattenberg, M. Wicke, Y. Yu, and X. Zheng, 2016, TensorFlow: Large-Scale Machine Learning on Heterogeneous Distributed Systems: Technical report.

Ahmed, A., A. Cosse, and L. Demanet, 2015, A convex approach to blind deconvolution with diverse inputs: 2015 IEEE 6th International Workshop on Computational Advances in Multi-Sensor Adaptive Processing, CAMSAP 2015, Institute of Electrical and Electronics Engineers Inc., 5-8.

Bharadwaj, P., L. Demanet, and A. Fournier, 2019, Focused Blind Deconvolution: IEEE Transactions on Signal Processing, 67, 3168-3180.

Carcione, J. M., D. Kosloff, and R. Kosloff, 1988, Wave propagation simulation in a linear viscoacoustic medium: Geophysical Journal, 93, 393-401.

Chen, T. Q., X. Li, R. Grosse, and D. Duvenaud, 2018, Isolating sources of disentanglement in variational autoencoders: 6th International Conference on Learning Representations, ICLR 2018 - Workshop Track Proceedings.

Cohen, T. S., M. Weiler, B. Kicanaoglu, and M. Welling, 2019, Gauge equivariant convolutional networks and the icosahedral CNN: 36th International Conference on Machine Learning, ICML 2019, 2019-June, $2357-2371$.

De Ridder, S. A., B. L. Biondi, and R. G. Clapp, 2014, Time-lapse seismic noise correlation tomography at Valhall: Geophysical Research Letters, 41, 6116-6122.

Eyre, T. S., D. W. Eaton, D. I. Garagash, M. Zecevic, M. Venieri, R. Weir, and D. C. Lawton, 2019, The role of aseismic slip in hydraulic fracturing-induced seismicity: Science Advances, 5, 1-11.

Garnier, J., and G. Papanicolaou, 2016, Passive imaging with ambient noise.

Goodfellow, I., Y. Bengio, and A. Courville, 2016, Deep learning: MIT Press.

Greydanus, S., M. Dzamba, and J. Yosinski, 2019, Hamiltonian Neural Networks.

Ilse, M., J. M. Tomczak, and M. Welling, 2018, Attention-based deep multiple instance learning: 35th International Conference on Machine Learning, ICML 2018, 5, 3376-3391.

Klys, J., J. Snell, and R. Zemel, 2018, Learning latent subspaces in variational autoencoders: Technical report. Kramer, M. A., 1991, Nonlinear principal component analysis using autoassociative neural networks: AIChE Journal, 37, 233-243.

Lumley, D. E., 2001, Time-lapse seismic reservoir monitoring: Geophysics, 66, 50-53.

Mattheakis, M., P. Protopapas, D. Sondak, M. Di Giovanni, and E. Kaxiras, 2019, Physical Symmetries Embedded in Neural Networks: arXiv:1904.08991 [physics].

Maxwell, S. C., J. Rutledge, R. Jones, and M. Fehler, 2010, Petroleum reservoir characterization using downhole microseismic monitoring: Geophysics, $\mathbf{7 5}$. 
Mordret, A., N. M. Shapiro, and S. Singh, 2014, Seismic noise-based time-lapse monitoring of the Valhall overburden: Geophysical Research Letters, 41, 4945-4952.

Nakata, N., and R. Snieder, 2012, Estimating near-surface shear wave velocities in Japan by applying seismic interferometry to KiK-net data: Journal of Geophysical Research: Solid Earth, 117, n/a-n/a.

Pevzner, R., V. Shulakova, A. Kepic, and M. Urosevic, 2011, Repeatability analysis of land time-lapse seismic data: CO2CRC Otway pilot project case study: Geophysical Prospecting, 59, 66-77.

Rickett, J., 2007, Estimating attenuation and the relative information content of amplitude and phase spectra: Geophysics, 72.

Robertsson, J. O., J. O. Blanch, and W. W. Symes, 1994, Viscoelastic finite-difference modeling: Geophysics, 59, 1444-1456.

Shabelansky, A. H., A. Malcolm, and M. Fehler, 2015, Monitoring viscosity changes from time-lapse seismic attenuation: Case study from a heavy oil reservoir: Geophysical Prospecting, 63, 1070-1085.

Wang, Z., M. E. Cates, and R. T. Langa, 1996, Seismic monitoring of CO2 flooding in a carbonate reservoir: Rock physics study: 1996 SEG Annual Meeting, 63, 1886-1889.

White, J. A., and W. Foxall, 2016, Assessing induced seismicity risk at CO2 storage projects: Recent progress and remaining challenges: International Journal of Greenhouse Gas Control, 49, 413-424.

Winkler, K., and A. Nur, 1979, Pore fluids and seismic attenuation in rocks: Geophysical Research Letters, 6, $1-4$.

Zaheer, M., S. Kottur, S. Ravanbhakhsh, B. Póczos, R. Salakhutdinov, and A. J. Smola, 2017, Deep sets: Advances in Neural Information Processing Systems, 2017-Decem, 3392-3402. 